\title{
ESTUDIOS BIOGRÁFICOS EN INSTITUCIONES EDUCATIVAS EN MÉXICO ${ }^{1}$
}

\section{- JOSÉ ANTONIO SERRANO CASTAÑEDA}

Universidad Pedagógica Nacional

\section{JUAN MARIO RAMOS MORALES}

Universidad Pedagógica Nacional

RESUMEN A manera de estado de conocimiento que va desde 2007 a 2017, los autores describen los modos en que opera la perspectiva biográfico-narrativa en los trabajos de investigación que los nativos del país han producido desde el campo en el sistema educativo mexicano. Exponen los tres ámbitos genéricos en los que se han desplegado deliberaciones: trayectorias de agentes en educación inicial y educación básica; trayectorias de agentes en educación media superior; trayectorias de agentes en educación superior; cada uno incluye posición en la posición. Los autores incluidos en este texto aprehenden de manera particular lo biográfico y lo enlazan con sus temas de reflexión o problemas de investigación. Más allá de la denominación de historia de vida, relatos de vida o biografía en el contexto mexicano hay un uso particular de la noción de trayectoria para dar cuenta de la escritura de la vida. Además de la diversificación de los asuntos en el sistema educativo, son variadas las fuentes teóricas o perspectivas que nutren las deliberaciones de los indagadores. Para la comprensión de lo biográfico los autores se apuntalan en diversos materiales provenientes de lenguas como el inglés, el francés o alemán, italiano y portugués. El texto finaliza con reflexiones sobre la orientación del campo, el lenguaje utilizado, las perspectivas en uso que se han desplegado para dar cuenta de la trama en que los sujetos viven su paso por la escuela.

Palabras clave: Biografía. Narrativa. Trayectorias. Sistema educativo mexicano. Estado de arte.

\section{ABSTRACT BIOGRAPHICAL STUDIES IN EDUCATIONAL INSTITUTIONS IN MEXICO}

As a state of knowledge that goes from 2007 to 2017, the authors de-

1 Los autores agradecemos el apoyo en la búsqueda bibliográfica de Sandra de la Rosa y Flora Ivón Jiménez, egresadas de la licenciatura en Pedagogía de la Universidad Pedagógica Nacional. 
scribe the ways in which the biographical-narrative perspective operates in the research work that the natives of the country have produced in the Mexican educational system. They outline three generic areas in which deliberations have been carried out: trajectories of agents in initial and basic education; trajectories of agents in upper secondary education; trajectories of agents in higher education. Each one includes position in the position. The authors, included in this text, show that they particularly apprehend the biographical and link it with their reflection themes or research problems. Beyond the denomination of life history, life stories or biography in the Mexican context there is a particular use of the notion of trajectory to account for the writing of life. In addition to the diversification of issues in the education system, the theoretical sources or perspectives that feed the deliberations of the inquirers are varied. For the understanding of the biographical the authors are nourished of diverse materials coming from languages like the English, the French or German, Italian and Portuguese. The text ends with reflections on the orientation of the field, the language used, the perspectives in use that have been deployed to give an account of the plot in which the subjects live their passage through the school.

Keywords: Biography. Narrative. Trajectory. State of the art. México.

\section{RESUMO ESTUDOS BIOGRÁFICOS EM INSTITUIÇÕES EDUCACIONAIS NO MÉXICO}

Como um estado de conhecimento que vai de 2007 a 2017, os autores descrevem as formas em que a perspectiva biográfico-narrativa opera no trabalho de pesquisa que os nativos do país produziram no sistema educativo mexicano. Descrevem as três áreas genéricas em que as deliberações foram realizadas: trajetórias de agentes na educação inicial e na educação básica; trajetórias de agentes no ensino médio; trajetórias de agentes no ensino superior. Itens genéricos que incluem posição na posição. Os autores dos textos revisados apreendem o biográfico e o vinculam aos seus temas de reflexão e problemas de pesquisa. Além das denominações história de vida, histórias de vida ou biografia, no contexto mexicano, há um uso particular da noção de trajetória para explicar a escrita da vida. Além da diversificação de questões no sistema educacional, as fontes ou perspectivas teóricas que alimentam as deliberações dos inquiridores são variadas. Para a compreensão do biográfico, os autores nutrem-se de diversos materiais em diferentes idiomas: inglês, francês ou alemão, italiano e português. 0 texto termina com reflexões sobre 
a orientação do campo, o idioma utilizado e as perspectivas em uso que foram implantadas para dar conta da trama em que os sujeitos vivem sua passagem pela escola.

Palavras-chave: Biografia. Narrativa. Trayectorias. Sistema educativo mexicano. Estado da arte.

¿La experiencia vivida?

Esa expresión es una tautología.

La experiencia siempre es vivida

Jean Grondin

Estado de arte, estado de conocimiento, estado de la cuestión son denominaciones que se le han dado en México - desde finales de la década de los años setenta y principios de los ochenta del siglo pasado- a la valoración de la producción sobre un tema, en tanto fase preliminar en el proceso indagador (JIMÉNEZ, 2009). Desde 1981, el Consejo Mexicano de Investigación Educativa ha promovido la elaboración de estados de conocimiento sobre el campo educativo. Si bien los investigadores han tomado criterios específicos de boca a boca, se ha diferenciado al estado de arte como el conocimiento de frontera de un campo a la manera de los handbook. El estado de conocimiento - o estado de la cuestión- es la búsqueda amplia de producción sobre un campo con delimitaciones temporales y geográficas que, con base en criterios específicos, tiende a la categorización de la información recabada (DALLE, 2005). Con base en éstas ideas emprendimos el presente trabajo que espera dar luz sobre cómo en México se ha realizado investigación en el campo biográfico-narrativo en el sistema educativo mexicano en el periodo establecido.

Este artículo, por un lado, se enlaza a una serie de reflexiones que hemos desarrollado sobre la constitución de los campos de conocimiento y sobre lo idiosincrático de los temas (BECHER, 2002), asuntos y modos de escritura que dan vida al ámbito biográfico. Además, asume en su confección las ideas que en Mexican educational system se han planteado para la elaboración de estados de conocimiento. Caracterizados como

[...] el análisis sistemático y la valoración del conocimiento y de la producción generadas en torno a un campo de investigación durante un periodo determinado. Esto permitiría identificar los objetos bajo estudio y sus referentes conceptuales, las principales perspectivas teóricas-metodológicas, tendencias y temas abordados, el tipo de producción generada. (RUEDA, 2012, p. 4)

En esta investigación retomamos la caracterización indicada. El trabajo da continuidad al expuesto en Serrano y Ramos (2014), donde planteamos los enlaces entre la cultura local y la configuración de las ciencias sociales en México para cogitar sobre el ámbito biográfico en educación en el periodo que va del 2003 al 2013. En el presente texto cambia la temporalidad y la selección de los textos. Primero, describimos las argumentaciones de los autores sobre lo biográfico vinculadas el ámbito educativo y, después, establecemos un balance general de lo expuesto.

No sobra señalar que los estados de conocimiento son una investigación bibliográfica para la construcción de conocimientos en una rama específica de saber y están orientados a la exposición del sentido que los autores analizados le dan al tema en cuestión. Tomamos a cada texto como una totalidad y buscamos en cada una de las partes las relaciones internas de producción de significado, como una manera de unir lo fenomenológico y lo her- 
menéutico. Es una manera de poner en acto el enunciado "Comprender, según Ricoeur, no es entonces, ante todo, dar un sentido, sino captar un sentido dado que se perpetúa más allá de su lugar inicial de enunciación" (FOESSEL, 2008, p. 33, cursivas del autor).

Para este trabajo, realizamos una búsqueda inicial en diferentes bases de datos sobre el tema de lo biográfico, nos apoyamos en palabras clave: "biografia", "enfoque narrativo", "historias de vida", "relatos de vida", "historias orales", "relatos de formación", "relatos de prácticas", "trayectorias escolares", "trayectorias biográficas", "trayectorias institucionales". La búsqueda, organizada en una base de Excel, arrojó 119 textos de diversa índole y de variadas formas de difusión². La prolijidad de documentos nos llevó a establecer criterios: informes con referentes concretos, informes elaborados por mexicanos durante el periodo indicado que estuviesen publicados (dado que eso indicaría algún efecto de circulación entre la comunidad académica) como libros, capítulos de libros y artículos en revistas especializadas. Por la extensión y características de publicación, no se incluyeron tesis de grado y de posgrado. Además, nos centramos en bibliografía que complementa la analizada en Serrano y Ramos (2014).

Los documentos encontrados nos permitieron afinar nuestro objetivo: inventariar, delinear las tonalidades sobre lo biográfico durante la década del 2007 al 2017 en informes de investigaciones con los criterios arriba expuestos. Metodológicamente procedimos al análisis del material con base en las siguientes preguntas: ¿cuál es el sentido propio que le dan a lo biográfico?, ¿cuáles son los autores que retoman en sus deliberaciones?, ¿cómo resuelven lo metodológico en sus indagacio-

2 La difusión, organización en el campo educativo aún son deficientes. Procuramos delimitar el trabajo y recoger la mayor cantidad de trabajos más reconocemos que este acercamiento no recupera toda la producción generada en la década indicada. nes?, ¿cuáles son los resultados que exponen? El corolario de esta tarea es el texto que el lector tiene en sus manos.

Creemos que los criterios de agrupación ya son un acto de violencia hacia el mismo texto. Empero, el estilo argumentativo seleccionado pretende dar voz a los autores con su particular talante. Al final, como en Serrano y Ramos (2014), planteamos algunas cuestiones que -en continuación con aquel y en particular a partir de la producción mostrada en este texto- nos dejaron en tanto deliberaciones sobre el movimiento del campo.

Hemos visto las ventajas de hacer cortes de caja en los campos de conocimiento en los que participamos y promovemos la misma estrategia en nuestros estudiantes. Material que dio cuerpo al libro Formación de profesionales de la educación: sujetos, reflexión y producción de saber (ORTIZ y SERRANO, 2016), en donde mostramos la costumbre sana de sabanear, de sublimar al voyerista que otea artículos y libros sobre el ámbito biográfico narrativo. Costumbre que lleva a posicionarse sobre lo producido y proyectar nuevas brechas $y$, en el camino, -fiel a la metáfora geográfica relativa a la noción de campo- percibir huecos, rendijas, hendiduras, oquedades, fisuras en el interminable camino de dar cuenta de la vida de los sujetos en contextos escolarizados. El lector encontrará una invitación para posicionarse $y$, tal vez, localizar filones que permitan el usufructo de algún yacimiento en el amplio terrero de lo biográfico-narrativo. Dada la reiteración de lo biográfico ligado a la noción de trayectoria, con base en ella organizamos los diferentes apartados. Para el análisis y exposición de los textos seleccionados tomamos como referencia el sistema educativo mexicano: trayectorias de agentes en educación inicial y educación básica; trayectorias de agentes en educación media superior; trayectorias de agentes en educación superior. 
Trayectorias de agentes en

\section{educación inicial y educación básica}

En este primer rubro encontramos investigaciones de diverso calibre, con una misma orientación, el trabajo biográfico alrededor de la idea de trayectoria, ya con énfasis en la trama individual o la trama ligada a la comprensión de prácticas. Hemos organizado el apartado en tres rubros: trayectoria de maestras y maestros; trayectorias subdirectores; trayectorias de supervisoras.

\section{Trayectoria de maestras y maestros}

Salazar y Sánchez (2016) investigan sobre estudiantes mujeres - de la Licenciatura en Educación Preescolar (LEP) con Tecnologías de la Información y la Comunicación diseñada por la Universidad Pedagógica Nacional (UPN)- que, simultáneamente, son trabajadoras en centros comunitarios y estancias infantiles del Gobierno del Distrito Federal, dedicadas a la atención en educación inicial y preescolar. Parten de las siguientes preguntas “¿cuáles son los procesos formativos que han vivido estas profesoras a través de su trayectoria profesional? ¿de qué manera se forman como docentes en la práctica? ¿Cuáles son los procesos formativos que viven como alumnas de la LEP?" (SALAZAR y SÁNCHEZ, 2016, p. 16). El estudio tiene un fin doble, tener información de la formación recibida y de la apropiación de la formación a partir de los relatos que ellas elaboran con indicaciones de las docentes. Fundamentan el trabajo en el campo biográfico-narrativo a partir de las Goodson (etapas en la vida), de ideas de Bourdieu (trayectoria), de Bolivar (enfoque biográfico), enlazan con ideas de Ricoeur (narratividad) para enmarcar pautas culturales y de Bajtin (lenguaje) en relación con el relato biográfico que las estudiantes construyen en el dispositivo de investigación. Para las autoras, las trayectorias consisten en acercarse a la vida personal y profesional. Metodológicamente la que bautizan como investigación experiencial: "abarca cualquier forma de reflexión oral o escrita que utiliza la experiencia personal en su dimensión temporal" (SALAZAR y SÁNCHEZ, 2016, p. 18).

Perciben a la narrativa, a la escritura de la historia de vida de cada estudiante, como un dispositivo para verse a sí mismas en lo que han sido y lo que son como mujeres con proyectos profesionales. En la escritura las estudiantes construyen su trayectoria. Las investigadoras asientan que la trayectoria es (caracterización directamente vinculada con los planteamientos de Bourdieu) "la serie de posiciones sucesivamente ocupadas por un mismo agente, o grupo, en un espacio que es cambiante en sí mismo" (SALAZAR y SÁNCHEZ, 2016, p. 19) que permiten comprender las vivencias de las profesoras en el trabajo que efectúan ante las demandas de la institución. En su construcción asumen las ideas de Goodson de etapas, como una forma de analizar los momentos y sus vericuetos para alcanzar su proyecto de devenir profesionales de la educación. Metodológicamente, inician con la escritura de la trayectoria profesional. Después, profundizan en la vida personal de las estudiantes, una manera de enlazar lo social con lo personal que favorece procesos reflexivos. Luego -con los escritos elaborados-, construyen categorías para dar cuenta de la visión del mundo de las estudiantes. Más adelante identifican cinco etapas.

Quezada (2015), en “Trayectorias formativas e historias institucionales en la configuración de identidades: el caso de los maestros de educación especial", indaga sobre los procesos, espacios, actividades y mecanismos con los que la escuela contribuye en la construc- 
ción de la identidad, dice, "la construcción subjetiva del sentido la realiza el actor desde su situación biográfica". Recurre a dos espacios escolares: la enseñanza de la historia en la escuela y el análisis de prácticas educativas para su enseñanza. En el abordaje conceptual recurre a aspectos socioculturales y territoriales sustentado en Giménez, Durkheim, Berger, Luckmann, Giddens; y en las nociones de habitus y ethos de Bourdieu para la construcción de identidades socioterritoriales. El acercamiento a la temática lo realiza, por un lado, desde la narrativa histórica con técnicas como el análisis del discurso, el análisis comparativo, el análisis semántico y el análisis político conceptual (p. 68) y, por otro, desde la etnografia con observaciones, entrevistas a profesores y a niños estudiantes, relatos biográficos (p. 6869). Finaliza con reflexiones sobre los trayectos recorridos por los profesores, en la enseñanza (sentidos, contenidos identitario, narrativa oficial, posturas, pasión o indiferencia, respeto o veneración) y los estudiantes (referentes adquiridos en hogares, comunidades, medios de comunicación); aspectos socioterritoriales que inciden en el proceso de configuración de identidades en los contextos escolares.

Quezada indica que, si bien, "en la conformación de identidades no puede hablarse de modelos preestablecidos que llegan a reproducirse e imponerse" (p. 95), en las trayectorias escolares de los alumnos -en particular en los dos espacios escolares estudiados por la autora (enseñanza de la historia, prácticas educativas)-, la escuela provee un modelo, uniforma sentidos y significados, genera y refuerza el sentido de pertenencia hacia orígenes y procesos comunes que no están aislados de los referentes adquiridos en otros contextos (familiares, sociales, comunitarios, medios masivos) a partir de lo cual "se apropian de una identidad socioterritoriale los niños y jóvenes estudiantes" (p. 94).
Por su parte, Toriz (2015) en "Transformaciones de la profesión docente como una institución y malestar en la escuela secundaria pública" indaga sobre la relación del malestar del profesor y la institución de la profesión docente. El abordaje teórico lo realiza desde las ideas de malestar, sociedad de conocimiento, psicología social y profesión con los recursos de Schvarstein, Castillo, Esteve, Berger y Luckmann, Dubet, Vela, Käes, Gaujelac. Elabora trayectorias del profesorado vía entrevistas a profundidad (Rubio y Varas) y la noción de trayectorias profesionales del profesorado (Bertaux). El trabajo realizado con las trayectorias de maestros de una escuela secundaria pública lo brindan elementos para mostrar las relaciones entre la institución de la profesión docente en dos momentos (años ochenta y noventa); compara la relación entre profesor y estudiantes, el cambio en los contextos escolares, institucionales (SEN), de la vocación docente y de las características de ambos sujetos.

\section{Trayectorias subdirectores}

Navarro (2015) dedica un amplio estudio para comprender la función directiva. Se apoya en la tradición biográfico-narrativa, para el caso recurre a un abanico amplio de información. El entramado que permite la construcción de los relatos biográficos adhiere a la noción de trayectoria un tesoro lingüístico particular "fuerzas conductoras, puntos de cambio, marca" (NAVARRO, 2015, p. 70, cursivas del autor). Además, recurre "al concepto de socialización", al de "liderazgo y dirección" y a los de "dominación y la legitimación de la autoridad" que le "permiten hermanar una mirada común e integrar un marco teórico para la interpretación y sustento de los relatos" (NAVARRO, 2015, p. 71, todas cursivas de la autora).

Con base en la idea de Bourdieu sobre trayectoria ("serie de posiciones sucesivamente 
ocupadas por un mismo agente (o un mismo grupo) en un espacio en sí mismo en movimiento y sometido a incesantes transformaciones", 1997 p. 82), Navarro incorpora la idea de itinerario para referirse a la cronología de las posiciones ocupadas por los sujetos. "Los itinerarios, en conjunto con los cambios y los motivos que lo originan, configuran el estudio de las trayectorias" (NAVARRO, 2015, p. 73). Para una mejor exposición de los itinerarios, la autora retoma cinco orientaciones conceptuales: la primera, "fuerzas conductoras... el principio generado del cambio de posición"; otro es el de "punto de cambio... sería el proceso que rompe la relación entre la secuencia previa y posterior al evento en cuestión" (NAVARRO, 2015, p. 74); un concepto más es el de marca, referido a lo que tiene efecto de constitución en el ámbito profesional; luego, introduce la noción de trayecto que sería aquello que los sujetos efectúan antes de la llegada a la dirección de la escuela, propone tres tipos de trayectos: el formativo, profesional y laboral; finalmente, la institución, advierte que ninguna trayectoria se realiza fuera del contexto, en tanto comunidad que da sentido a la identidad lograda. Para el análisis del material, Navarro recurre a diversas tradiciones en ciencias sociales: socialización, Berger y Luckmann; liderazgo, dirección, Bolívar; dominación, Weber.

El trabajo lo ubica en la investigación narrativa, modalidad de la investigación cualitativa que tiende a trabajar desde la perspectiva biográfica con los relatos que los sujetos presentan en las entrevistas. Para la autora, la entrevista a profundidad es la vía regia para la construcción de los datos de las experiencias, vivencias que mediante el recuerdo acceden al presente, no sin antes pasar por la criba de la subjetividad -inherente a los individuos que han sido marcados por el mundo cultural, efectos de la socialización- que lleva a la elaboración continua del mundo que les rodea. Lo que la autora obtiene en los relatos es la conexión establecida por la memoria y el recuerdo pues "los relatos biográficos no son idénticos a las vivencias sino semejantes." (NAVARRO, 2015, p. 110). Además, reconoce el lugar del establecimiento de relaciones cronológicas. En el proceso de indagación, la entrevista formó parte de otros tipos de instrumentos: un cuestionario de identificación de participantes, cuatro guiones de entrevista a ser cubiertos en cada integrante, una bitácora de observación para las entrevistas. Entre diversas aportaciones, concluye que el sistema educativo no tiene una definición clara del puesto y que son los sujetos concretos que le dan sentido al rol institucional.

\section{Trayectorias de supervisoras}

Rivera (2015) recurre a los relatos de prácticas desde las nociones planteadas por Bertaux al respecto. El trabajo de Rivera -Aportes teórico-metodológicos para el estudio de las prácticas en contextos institucionales: el caso de las supervisoras en educación preescolar- toma como objeto de estudio el caso de las supervisoras de preescolar para trazar sus prácticas en contextos institucionales. El abordaje teórico proviene de autores como Berger y Luckmann, Dubet, Etkin y Schvarstein, Enríquez y Bourdieu. En la noción de trayectoria recurre a De Certeau y para el relato de práctica se apuntala en Bertaux. Rivera señala que una de sus preocupaciones básicas "estaba relacionada con el interés de estudiar las prácticas sin reducirlas a su dimensión subjetiva o a una colección de relatos biográficos de carácter fenomenológico aislados del campo en el que se producen y adquieren sentido" (RIVERA, 2015, p. 304-305). Para los relatos de práctica, Rivera se nutre de entrevistas a profundidad a fin de mostrar "cómo interpretan, interiorizan y significan las supervisoras los objetos, fines 
y medios que otorgan algún tipo de significado a la función de supervisión, con base en los patrones y modos de institucionalización configurados a nivel histórico" (RIVERA, 2015, p. 305). En las conclusiones indica que el trabajo -vía relatos de práctica- le brindó elementos para señalar que la supervisión "forma parte de un entramado institucional y distinguir a la supervisión como parte de un campo de relaciones más amplio como la educación básica. A las supervisoras como sujetos sociales... con una trayectoria rural e individual" (p. 317) que en su práctica "condensan la multiplicidad, heterogeneidad y pluralidad de objetos, finalidades, formas de institucionalización, recursos y significados sobre la supervisión" (p. 318). Reconoce que las trayectorias son el "resultado de un largo proceso de acumulación y apropiación singular de conjunto de experiencias diversas, constituye un elemento importante para comprender estas diferencias" (RIVERA, 2015, p. 318).

\section{Trayectorias de agentes en educación media superior}

A nivel mundial, el sector de educación media superior es uno de los menos estudiados y donde se concentran buena parte de los problemas a atender, por lo menos en México. El apartado expresa la deserción de estudios en el rubro y el poco eco de la perspectiva biográfico-narrativa en educación. El apartado muestra dos temáticas: trayectorias de estudiantes, trayectorias de jóvenes y trayectorias de madres adolescentes.

\section{Trayectorias de estudiantes}

En el texto coordinado por Guzmán y Saucedo (2007) encontramos diversos trabajos enfoca- dos al acercamiento a jóvenes, en particular de educación media superior (bachillerato) que recuperan la experiencia de los sujetos con propósitos y perspectivas variopintas. Al trabajar con los textos, identificamos tres de ellos que atienden al tema de lo biográfico desde los descriptores indicados. Por un lado, Saucedo (2007) orienta su indagación al abordaje del papel de la escuela desde la experiencia de los estudiantes mediante la elaboración de relatos de vida. Acude a entrevistas a profundidad para trabajar la narrativa bajo la perspectiva de Bruner y la construcción de relatos de vida planteada por Becker; además se apoya en autores como Ochs y Capps, Wertsch, Portelli e Irwin para el abordaje y organización de las narraciones y los relatos. Trabajó con jóvenes y sus padres pertenecientes a un contexto de "hogares ferrocarrileros". Organiza los relatos a partir de dejar o regresar a la escuela y considerar el estudio como condición y proyección de vida. Encuentra "respuestas diversas hacia la escolaridad" (p. 40), los vínculos con los contextos social y escolar -conflictos y correspondencias-, el valor de la escuela; refiere la reflexión de los jóvenes en torno al abandono de los estudios y sus expectativas de vida laboral y la reescritura permanente de sus vidas y de la escolarización; que la llevan a discurrir sobre la necesidad de "pensar las trayectorias escolares como recorridos, como construcciones que se van dando paso al tiempo y para los cuales no hay sentidos prefigurados del todo ni puntos de arribo seguros" (p. 41).

Por su parte, Velázquez (2007) quien se preocupa por la experiencia de ser estudiante en preparatoria. Perfila su trabajo desde la noción de trayectoria de Bourdieu. Caracteriza la trayectoria ideal y, vía los relatos, expone los hallazgos en torno a los giros en las trayectorias escolares interrumpidas: trayectorias unirre- 
gresionales, trayectorias multirregresionales, trayectorias regresionales. Además de recuperar los relatos que abordan la experiencia de ser examinados al ingreso y las dificultades en el proceso de adaptación en este nivel educativo. En el análisis se apoya en Machado Pais, Craine, Romani, Lansén. Dubet y Martuccelli, Jackson, Coulon, entre otros. En las reflexiones finales indica: "hoy se hace necesario entender la diversidad de los modos de ser estudiante, lo que permitirá comprender que la convivencia entre diferentes estudiantes con diferentes formas de sentir y valorar, diferentes habitus, diferentes trayectorias" (p. 66).

Merino y Ramírez (2007), en "la construcción subjetiva de la identidad en las autobiografias de estudiantes adolescentes", se proponen analizar narrativas autobiográficas de jóvenes de preparatoria de la UNAM, en particular, "su proceso de desarrollo psicosocial" (p. 171). Sostiene su trabajo con referentes teóricos sobre la identidad, las tecnologías del yo, (Erikson, Freud, Foucault), además se apoya en Anna Freud para aproximarse al tema de la adolescencia y a Blos en las "estaciones de paso" (hitos en el desarrollo progresivo) y Piaget, Inhelder, Kohlberg, Elkind, entre otros. A partir de estos referentes acomete "la tarea analítica interpretativa "atrapar» al yo autor de la narrativa autobiográfica" (p. 173). En las autobiografias encuentra diversas posiciones y zonas en torno a la identidad de los estudiantes como: negativa, confusión, difusión, crisis y conquista. Los aspectos abordados en las narrativas, dice, son "Los hilos, los tintes y colores, las formas y texturas de ese tejido que es la autobiografia" (p. 190) y que los estudiantes bordan con los trayectos recorridos en entornos familiares, escolares y culturales. Además, el trabajo con las narrativas le permitió vislumbrar procesos alrededor de la identidad de género; la lucha por su identidad; aspectos de autoestima y desempeño académico; la prolongación de la adolescencia entre otros y cuestionarse sobre la atención escolar-académica-de desarrollo que se da a la condición y problemática adolescente en los sistemas educativos.

\section{Trayectorias de jóvenes}

Franco (2015) orienta su trabajo a identificar las "marcas identitarias [de los estudiantes] a partir de procesos de apropiación del espacio escolar como un espacio público constitutivo y referencial" (FRANCO, 2015, p. 37) a partir de dos referentes: trayectoria escolar y pertenencia. Asume la noción de identidad desde los planteamientos de Dubar, Dubet y Mantuccelli y Giménez. Metodológicamente, apela a la narrativa biográfica desde Giménez y a la etnografia planteada por Geertz y Thompson, Woods. Toma como referente de estudio un bachillerato en Tepeojuma, Puebla, un municipio en el que la migración (nacional o internacional) se convierte en una forma o proyecto de vida para los jóvenes. Concluye que los sujetos entienden la lógica de ser estudiantes por el recorrido entre los niveles de escolarización. Asimismo, los sujetos advierten los "ejes rectores para lograr el deber ser", la disciplina y el éxito (p. 62). Añade que "es el espacio escolar donde los sujetos aprenden "desde la producción de sentido, la trama social de lo que se va llegando a ser desde el proceso de formación escolarizado" (p. 63).

\section{Trayectorias de madres adolescentes}

García (2016) apuntala su indagación sobre las madres adolescentes en contexto de pobreza en el "método biográfico y curso de vida". Para ello, se planta ocho preguntas, la primera de ellas es “¿Cuáles son las distintas trayectorias sexuales reproductivas que recorren las ado- 
lescentes hasta que tienen el primer hijo?" (GARCíA, 2016, p. 21). Pregunta que le dirige su atención al tratamiento de los asuntos relativos a la trayectoria escolar y a los itinerarios laborales.

Para dar cuenta de su objeto de estudio, García emplea tres metodologías “i) investigación de campo y documental... ii) encuesta aplicada a la población... iii) 19 entrevistas biográficas a madres y adolescentes" (GARCíA, 2016, p. 21). La autora seleccionó a mujeres incluidas en el sector de alta marginación que se embarazaron antes de los dieciocho años. Para explicar desde el punto de vista social, construye la categoría de marcadores de vulnerabilidad para dilucidar "las condiciones de desventaja y precariedad que se encontraron en las biografias de las madres adolescentes" (GARCíA, 2016, p. 20). En el tema de la vida sexual y reproductiva elabora la categoría de trayectoria sexual reproductiva. Con ella descifra "la secuencia de eventos relacionados al embarazo y los significados diferenciados que éste tuvo en los embarazos de las adolescentes" (GARCíA, 2016, p. 20).

De entrada, la narrativa devela la subjetividad de las mujeres entrevistadas dado que "recuperan y articulan los eventos y procesos que forman parte de la trayectoria sexual reproductiva... considera, además de la secuencia de eventos, los significados que las mujeres adolescentes elaboran en torno a ellos" (GARCíA, 2016, p. 70). Sirve como marco a sus afirmaciones los puntos de vista desplegados por las teorías de la vida cotidiana (Berger y Luckman; Heller; Goffman; Garfinkel; Shutz; Wolf). En su conjunto, los autores coinciden en asentar que la vida cotidiana es el lugar de la continuidad/ discontinuidad del mundo social, o en su versión social el espacio de la reproducción social de los hombres particulares.

El día a día es el territorio de la configuración de "la reproducción social a través de sus prácticas, valores, creencias, aspiraciones y necesidades" (GARCíA, 2016, p. 68). Desde tales barruntos, la autora rubrica su adscripción al método biográfico, revela las marcas de adscripción y la relación entre el mundo micro y macro social. La ventaja del método es que “permite acceder al sentido local de la vida, que de ningún otro modo es posible reconstruir, porque la biografía permite el acceso a esos cuadros culturales dentro de los cuales entran en acción el conocimiento del otro y de sí" (GARCíA, 2016, p. 65). Recurrir a lo biográfico es dar cuenta de las formas de edificación del yo y mostrar "las valoraciones que la persona hace de estos acontecimientos y de su propia vida. Se trata de un retorno al sujeto y a los significados" (GARCíA, 2016, p. 66).

Entonces, lo biográfico es un recurso para construir las trayectorias -escolar, laboral, familiar, de pareja- y develar en los relatos a quienes acompañan, a la interrelación de las personas con la familia, los pares y otras personas quienes colaboran en la organización de patrones y vínculos. Para García

[...] el concepto de trayectoria supone la noción
de agencia, ya que no necesariamente las per-
sonas se apegan a las reglas y expectativas de
su grupo, por eso no todas las personas que
comparten un momento sociohistórico tienen
el mismo curso de vida... es un proceso comple-
jo en el cual se articula un sistema de dispositi-
vos institucionales - familia, escuela, trabajo-
y procesos de socialización... en el que intervie-
ne la subjetividad... en el caso de la trayectoria
importan el grupo social de origen, el nivel de
educación alcanzado, el tipo de trabajo al que
se accede con él y la valoración social del mis-
mo. (GARCíA, 2016, p. 67)

De lo arriba expresado se desprende que el paso, el tránsito y el cambio de la trayectoria da cuenta de las transiciones. Transiciones y trayectorias están conectadas y las primeras no se entienden si no se toma en cuenta el grupo social de pertenencia. Por ejemplo, dos 
mujeres podrían ser madres a la misma edad y desertar de sus estudios en el mismo periodo, pero "lo que signifique la maternidad para una y para otra será diferente de acuerdo a su posición en la estructura social" (GARCíA, 2016, p. 67). Caracteriza a los argumentos de la autora el alejamiento de la noción de proyecto de vida que "implica un tipo de racionalidad más compleja y de proyección a futuro que no es común en este contexto" (GARCíA, 2016, p. 74), su lugar lo ocupa la idea de expectativa. García analiza la biografía bajo "tres mediaciones entre la estructura y las acciones del sujeto: las relaciones familiares, el binomio escuela trabajo y la relación de pareja" (GARCíA, 2016, p. 75).

\section{Trayectorias de agentes en educación superior}

El sector de educación superior ha recibido en el país atención especial por los grupos de investigadores. Los temas se han diversificado, sin que exista lejanía de los autores o nociones que constantemente aparecen en el medio académico. Los temas que en la producción resaltan son: trayectorias de científicos; trayectorias de académicos en educación superior; prácticas de institucionalización; trayectorias estudiantes de educación superior; trayectorias de profesionales de la educación.

\section{Trayectorias de científicos}

Montiel (2014) se interroga sobre "las condiciones en que se construyen trayectorias académicas de prestigio" (p. 23) de científicas que participan en el desarrollo de conocimiento de frontera en tres instituciones pertenecientes a la Universidad Nacional Autónoma de México: el Instituto de Biotecnología (IBTUNAM); el Centro de Radioastronomía y Astrofísica (CRyA-UNAM); el Instituto de Neuro- biología (INB-UNAM). En especial, se plantea las preguntas “a) ¿cuáles fueron los recursos con que lograron construir una trayectoria de prestigio?; b) ¿cuáles fueron las condiciones que les permitieron una participación consolidada en la comunidad científica nacional e internacional?" (MONTIEL, 2014, p. 28). En la investigación se recurre a lo biográfico como la exposición de los itinerarios acaecidos por los agentes en tanto cumplen el rol de científicas y su relación con la configuración de las instituciones en donde laboran y la configuración del campo de conocimiento en donde realizan su producción.

Para dar cuenta del recorrido de cada una de las científicas, expone algunas consideraciones vinculadas: la formación en investigación científica, las comunidades académicas que cobijaron la formación, el contexto institucional particular, la inscripción en diversos grupos que promovieron la participación en redes y circuitos de producción, la configuración personal de estilos de liderazgo, la dirección de investigación y su participación en la institucionalización de los campos de conocimiento.

En su investigación sobre mujeres académicas, construye los tres casos con base en cuatro estrategias. Por un lado, utiliza la entrevista, desde la tradición etnográfica, como un vehículo para obtener enunciados que en la asociación de ideas producen temas de conversación relativas a su realidad y la manera en que perciben su inserción en las condiciones instituciones de las cuales son elemento rector para la producción de saber científico. La segunda estrategia fue recurrir a la revisión del Currículum Vitae único (CVU) que se encuentra en la base de datos del Conacyt en la que se registra la trayectoria académica de los investigadores mexicanos como forma de dar cuenta de la producción, los espacios formativos, la producción científica, los agentes y 
lugares de producción científica, así como los diversos reconocimientos recibidos. El CVU es la "oficialización de un discurso sobre sí mismo" (GARCIA SALORD, 2010: 5, citado en MONTIEL, 2014, p. 27). En tercer lugar, consultó otras bases de datos asociadas al registro de la producción científica de los académicos en México $y$, finalmente, los documentos institucionales que proporcionaron más datos sobre el recorrido efectuado en la institución que les permitió llegar al lugar en donde en estos momentos se localizan en la producción científica.

En tanto noción, "la trayectoria alude a los tramos que podemos reconstruir... identificando un recorrido posible y ofrece la descripción de la dimensión temporal de las prácticas, permite ver el ritmo y la duración de un proceso (continuidad, discontinuidad, intervalos)" (GARCIA SALORD, 2001: 5 citado en MONTIEL, 2014, p, 29). Para la autora, en la presentación de trayectorias se desvela el capital cultural, simbólico, social, económico, político, nociones vinculadas directamente a Pierre Bourdieu.

Del análisis efectuado a las trayectorias la autora construye tres configuraciones -una continuidad en formación científica, la apertura y construcción de un campo, una participación consolidada-. Concibe a la configuración como al "entretejido dinámico que implica una síntesis, a partir de nuevas integraciones como expresión de prácticas sociales, en las que confluyen no sólo contextos sino también múltiples construcciones de sujetos" (MONTIEL, 2014, p. 32).

En la exposición de las trayectorias muestra parte del registro de entrevista y de las condiciones objetivas de su producción, textos, proyectos, titulados. Al concluir advierte que las trayectorias se edifican ante la tensión con el contexto institucional, las políticas institucionales que movilizan a las universidades y el interés del sujeto por el campo.
En continuidad con la temática, Izquierdo (2015) coloca la mirada en la identidad académica de científicos soviéticos inmigrantes en México en la década de los noventa. El texto se centra en la exposición del abordaje teórico-metodológico de la investigación en la que pretende, por un lado, trazar los procesos de formación y construcción del pensamiento en torno a la ciencia soviética; por otro lado, esbozar el trabajo académico de los científicos en los dos países y las representaciones de su trabajo en el mundo de la ciencia. Muestra el origen del interés de la autora al objeto de estudio y su construcción: "la identidad [académica de los científicos soviéticos] y el enfoque biográfico" (IZQUIERDO, 2015, p. 128). Esboza el acercamiento al estudio de migraciones de científicos $y$, en particular, a trabajos sobre identidad académica realizados desde la dimensión biográfica, las trayectorias, los procesos de socialización e institución con la lectura de trabajos de García, Grediaga, Landesman, Hickman, Parra, Fortes, Lomnitz, Chavoya, Yurén, Navia, Saenger.

En particular, la autora se arroga el enfoque biográfico a través del relato de vida planteado por Bertaux para, vía el método cualitativo interpretativo, "tener acceso a la memoria de los científicos migrantes" (IZQUIERDO, 2015, p. 134) y "realizar el análisis de la información" (IZQUIERDO, 2015, p. 140). Además, se apoya en argumentos de Landesmann, Hickman, Parra, Marinas y Santa Marina. Izquierdo trabaja con quince científicos inmigrantes en México provenientes de la ex URSS. Con la entrevista accede -vía las narrativas de los entrevistados- a los relatos de vida organizados en seis ejes: "orígenes sociales; la socialización y la formación en la ciencia; el trabajo científico en la ex URSS; el proceso migratorio; el trabajo académico en México y las representaciones de ser un científico en México" (IZQUIERDO, 2015, p. 139). 


\section{Trayectorias de académicos en educación superior}

Romo y Cruz asumen las ideas de ethos, de Bourdieu; grupo e individuo, de Geertz; identidad de Dubar y Hall para efectuar un estudio comparativo con el uso de historias orales entre las carreras de Turismo y Psicología en la Universidad de Guadalajara. Asumen los supuestos de Bertaux sobre las historias orales: “Este abordaje posibilita estudiar un fragmento particular de la realidad socio-histórica y trabajarla como objeto social" (ROMO y CRUZ, 2015, p. 44). Se apoyan en la entrevista como estrategia de recolección de datos, sin mencionar las fuentes o tradiciones al respecto. Lo biográfico es asumido como técnica, en sus palabras "Utilizamos la técnica de relatos de vida porque nos interesa comprender tanto los procesos de interrelación entre los sujetos como los significados que construyen y se expresan en lo cotidiano, en la práctica profesional del académico." (ROMO y CRUZ, 2015, p. 45). Retoman, asimismo, los autores que han trabajado en México el asunto de las trayectorias de grupos institucionales para establecer tipologías de profesores integrantes de la institución, para el caso de los psicólogos: "fundadores", "los de afuera", "los de la vieja guardia", "psicólogos udegistas". No se asume la misma tipología para exponer los datos de los profesores de turismo.

Romo (2015), en el texto "La memoria en la reconstrucción de culturas académicas. Relatos de vida y arribo de la primera psicopedagogía a la Universidad Autónoma de Nuevo León [UANL]" hace un acercamiento a los aspectos teórico-metodológicos y el análisis de los relatos de vida y el vínculo de la memoria individual-colectiva. Traza la vida familiar, las migraciones (exilio y situación sociopolítica en su país de origen), la vida profesional y el contexto de la carrera de pedagogía en la
UANL. Conceptualmente, la autora acude a la concepción de relatos de vida de Bertaux y Geertz y a la noción de historizar de Ricoeur para remitirse a la memoria y sus procesos de constitución. Esboza los cruces, cambios, sentidos de las líneas temporales narradas por la entrevistada; los sentidos de sus historias (institucionales, grupales) que pone en juego los procesos de constitución de los sujetos a partir de los relatos biográficos -construidos a partir de la recuperación de la memoria- en tres momentos: el contexto histórico y el lugar de la mujer en el campo educativo; los aspectos teórico-metodológicos, el habitus académico y el trabajo con los datos empíricos desde la hermenéutica y la perspectiva biográfico narrativa; la exposición, interpretación y análisis de la narrativa de la entrevistada en torno al papel del contexto en la trayectoria académica.

González (2015) trabaja con relatos autobiográficos de investigadoras mexicanas con la finalidad de exponer "los elementos y momentos del contexto que las empoderaron para decidirse a incursionar en el plano paradigmático (filosófico-epistemológico) de la construcción teórica de lo educativo (GONZÁLEZ, 2015, p. 181). En su texto exhibe la influencia del pensamiento -Kant, Hegel, Rousseau, entre otros- que, de alguna manera, "excluye a las mujeres de su capacidad racional" (p. 183); y los argumentos metodológicos asumidos, la adhesión a la hermenéutica sin buscar metarrelatos o verdades generalizables y a los aspectos biográficos desde los planteamientos de Bonolio y Bertaux con las propuestas de la entrevista biográfica y los relatos de vida. En su trabajo elige cuatro investigadoras en el campo de la educación a partir de una consulta a investigadores internacionales e investigadoras nacionales, para trabajar entrevistas a profundidad y recuperar narrativas autobiográficas. En el análisis elabora categorías y las relaciones entre ellas. Identifica 
momentos clave en las vidas de las entrevistadas y agrupa las trayectorias en temporalidades -familiares, institucionales, geopolíticas, teóricas-, contrasta las narrativas con los curricula vitarum, emplea herramientas teóricas para el análisis de la información.

En el texto la autora expone sus hallazgos en las temporalidades planteadas, a partir de las cuales traza la influencia de los contextos en cada etapa -madres, padres, escolarización, el momento histórico, el entorno geopolítico-. Concluye con reflexiones en torno a la estrategia metodológica utilizada y el corpus "de información significativa para responder mi pregunta de investigación" (GONZÁLEZ, 2015 , p. 216). Asimismo, indica que, la estrategia metodológica utilizada le permite "evitar la comparación cuantificativa de acontecimientos" y centrarse en "el análisis diacrónico/sincrónico de la información y la contrastación de los hallazgos contra aspectos contractuales de las trayectorias" (p. 216).

Por su parte, Perales y Jiménez (2011) argumentan la pertinencia para estudiar las historias de vida de los docentes desde Bolívar, Eisner, Jackson y Fernández. Asumen la idea de habitus de Bourdieu. Indican la conveniencia de colocar en el centro de la discusión el punto de vista de los docentes para comprender tanto las condiciones del contexto educativo como para "abrir posibilidades para reformar, reestructurar y repensar la enseñanza" (PERALES, JIMÉNEZ, 2011, p. 143). Metodológicamente recurren a planteamientos de diversos autores para mostrar dos estrategias de escritura: el relato y la historia de vida. En ambos casos, exponen las narrativas de docentes que trazan el recorrido, así como el habitus, disposiciones y relaciones intersubjetivas, que siguieron en su elección y vínculo con el campo educativo. Dan cuenta de la forma en que en los relatos o historias de vida individuales es posible vislumbrar la generalidad social.
Güelmes (2015) emprende la tarea de "explicar y comprender" (p. 97) a los maestros de educación especial. Se centra en sus características, la elección de carrera, la forma como se constituyen y se autorrepresentan. Asume la perspectiva biográfica con el propósito de, a partir de sus historias de vida, dar voz a maestros especialistas en educación. Recurre a entrevistas y se apoya en los planteamientos de Weber, Stalamacchia, Coninck y Godard, Balán y Jelin, Bertaux con el propósito de "dar cuenta de esa historia de formación" (GÜELMES, 2015, p. 101) como maestros en educación especial de sordos y ciegos, vinculada a la historia institucional, la historia vivida previa a la carrera y la historia en el contexto familiar. En las historias presentadas, los entrevistados abordan los antecedentes de la medicina y de la educación especial a mediados del siglo XX para la conformación de estructuras curriculares, perfiles, los compromisos y formación de sus maestros, la ausencia del ejercicio práctico en el proceso formativo $\mathrm{y}$, en general, el trato secundario a la formación de maestros especialistas en la educación de sordos y ciegos por el énfasis en la formación de maestros para la educación de anormales mentales.

\section{Prácticas de institucionalización}

Zamora (2015), por su parte, se ocupa de elaborar relatos de vida con base en los planteamientos de Bertaux para abordar las "prácticas de institucionalización de la investigación educativa" ( $p .221$ ) en unidades de la Universidad Pedagógica Nacional. Trabaja con diez profesores distribuidos en tres sedes UPN: Ajusco, Mexicali y Pachuca. Para el análisis de la información construye categorías teóricas desde la perspectiva de autores como Berger y Luckmann, Bourdieu, Schvarstein y de Certau, a partir de los cuales elabora las trayectorias profesionales de los informantes. Aborda las 
construcciones socio-históricas, las tensiones entre trayectorias profesionales e historia socio-institucional y la relación de la trayectoria profesional con la historia institucional.

En el cierre de su texto, Zamora señala que las trayectorias de los académicos no siguen un recorrido lineal, el entramado contractual -familia, social, laboral, institucional-y las formas de apropiación de los sujetos las convierten en trayectorias complejas ajustadas a las situaciones que enfrentan. Reconoce, además, aspectos particulares en las trayectorias de los sujetos que conforman la actividad y prácticas de investigación en la UPN que remite a dos temporalidades: de génesis y subsecuentes (fundacionales, de desarrollo y de cambio); e, indica, las "trayectorias se decantan singular e institucionalmente conforme a la carrera académica emprendida por cada sujeto" (p. 255). El trabajo con trayectorias y con la identificación de tramas significativas, dice Zamora, "ayudan a ubicar a nuestros académicos como sujetos sociales singulares" (p. 256).

En el mismo sentido trabaja Hickman (2015) al abordar las trayectorias y procesos de institucionalización. La autora se enfoca a trabajar con académicas de la Universidad Veracruzana para vincular la trayectoria de un grupo con una disciplina particular, la psicología y su institucionalización. Para ello, recurre a diversas nociones y autores. En torno a la instucionalización recupera a Enriquez y Brunner; a Charlie y De Certau al hablar sobre dimensiones simbólicas y culturales. El mismo De Certeau le aporta elementos para trabajar las trayectorias y, metodológicamente, Bertaux le brinda la noción de relato de vida para privilegiar "la narrativa, la interpretación y la descripción de un fragmento de vida de los sujetos en situaciones institucionales diversas" (p. 266). A lo largo del texto, la autora aborda diversos momentos en las trayectorias de los sujetos articulados a los momentos institucionales; desvela encuentros, vínculos, rupturas, la fundación y legitimación de la carrera de psicología, así como estrategias de formación, socialización, difusión. En el cierre de su trabajo, señala el abordaje de los procesos de institucionalización e identifica cómo los trayectos e identidades de los sujetos son atravesados por aspectos subjetivos, simbólicos e imaginarios y "marcan de múltiples formas los modos en que ciertas 'realidades' (sociales, políticas, universitarias, entre otras) se configuran" (p. 291).

\section{Trayectorias estudiantes de educación superior}

Serrano y Ramos (2017b) sostienen que la relación entre lo biográfico y lo narrativo es eco de los giros que se han planteado en las ciencias sociales: lingüístico, cotidiano, pragmático, hermenéutico, afectivo, que bosquejan nuevas formas de superar los dualismos (individuo/ sociedad, naturaleza/cultura, entre otros). En su conjunto, reivindican al sujeto como agente que activamente participa en el devenir social, en la construcción de sentido para otros y para sí mismo. Al mismo tiempo, el enlace de lo biográfico y narrativo transvasa argumentaciones de tradiciones emparentadas con las preocupaciones del interaccionismo simbólico, el pragmatismo, la fenomenología y la hermenéutica, tales como: el mundo simbólico, lo prerreflexivo, la historicidad de la experiencia, el diálogo interno en el sujeto de la experiencia (SERRANO, et al, 2015). Lo biográfico deja de ser cuestión metodológica, para los autores se trasmuta en una forma de mirar los asuntos humanos.

En los asuntos biográficos resulta central el vocablo experiencia, retomado desde los planteamientos deweyanos (SERRANO, 2013; SERRANO y RAMOS, 2016, 2017a), como construcción histórica de lo acaecido en el mundo 
de vida. Los autores se valen de otras nociones como saber, sujeto, reflexión para dar cuenta del material empírico producido al tomar como objeto de trabajo las trayectorias de estudiantes de educación superior y los procesos de formación de docentes (SERRANO, 2014). Articulan en ambas preocupaciones la idea de que el yo en el proceso formativo se configura en el posicionamiento diferenciado de la trayectoria que construye al querer concretar el imaginario planteado en las circunstancias sociales e históricas en las que transcurre su vida (SERRANO, 2016a; SERRANO y RAMOS, 2014, 2017b; RAMOS, 2016; RAMOS y SERRANO, 2017).

Asumen que el sujeto es activo al tomar para si su proyecto formativo y se posiciona de variadas formas ante el entorno cultural. Si bien es cierto que lo institucional constriñe, también es el hecho de que el sujeto dispensa un sentido especial, una vida propia a las instituciones sociales (SERRANO, RAMOS, 2016; SERRANO, RAMOS, 2017c). Principio valido para dar cuenta de las relaciones entre los sujetos y los campos de conocimiento: en la biografía individual se localizan las formas de organización de los campos académicos. Son los sujetos concretos que proponen temas, modos argumentativos, adhesiones a paradigmas y proyectan problemas que se convierten en asuntos del campo de conocimiento (SERRANO y FURLAN, 2011; SERRANO, 2011).

Los autores han enlazado la trama de lo biográfico y lo narrativo en su modalidad de intervención para favorecer proceso de formación de docentes con el fin de generar procesos de mejora en las prácticas educativas. Se posesionan de la teoría de la indagación de Dewey para favorecer procesos reflexivos de innovación educativa (SERRANO, 2009; SERRANO, 2012b), para catapultar el saber del docente como vía para reflexionar sobre su lugar social en tanto educador e indagador de su saber sobre lo escolar (SERRANO, 2012a, 2014). Sos- tienen una perspectiva artesanal para la documentación de la experiencia de los sujetos: la entrevista como un saber de implicación que tiende a la producción de sentidos, del lado del entrevistador y del entrevistado (SERRANO, 2016b). La perspectiva descrita ha permito al grupo de trabajo asumir los argumentos arriba esbozados en diversas producciones de tesis de maestría y doctorado.

\section{Trayectorias de profesionales de la educación}

Torres (2011) aborda la transfiguración del pensamiento autobiográfico de la individualidad a la escritura y a compartir con los otros el ser, el estar con el mundo. La autora recurre al trabajo realizado en un seminario con estudiantes de posgrado (UPN y UNAM) para dar cuenta de la forma como, vía la escritura autobiográfica, los participantes, incluida ella misma, se posibilita la mirada hacia la propia vida a través de la asunción "del pasado y el futuro por el presente" (TORRES, 2011, p. 252).

La autora enlaza ideas en torno a la memoria, la escritura autobiográfica, con un esquema tripartita sobre el íncipit (comienzos, principio o inicio), la ruit (itinerario) y el exit (conclusión). Sostiene el papel del lenguaje en el trabajo autobiográfico para que el sujeto se conozca a sí mismo y resignifique los escenarios vivenciales. A partir de las nociones de narración, experiencias, tiempo, desvela las maneras en que los sujetos (profesionales de la educación) tejen, vía las autobiografías, "recuerdos, percepción, voluntad y deseo" (TORRES, 2011, p. 258). Nutre sus tesis con la exposición de un mosaico de extractos autobiográficos escritos por los participantes del seminario del que desprende diversas posiciones, decisiones que se juegan en la profesión educativa (docentes, maestros, universitarios). 
Torres (2012) toma como objeto de reflexión la trayectoria de una formadora de formadores para analizar de los tiempos, espacios y territorialidades en su autobiografía. Recupera la idea de escrito autobiográfico de Kaës - ligado a la tradición institucional- y las nociones de territorio-espacio las trabaja con las ideas de Ricoeur y Perrot. Destaca aspectos de la ruralidad del sujeto de estudio, "su participación política como espacio de articulación gremial y cultura en la Coordinadora Nacional de Trabajadores de la Educación y la Coordinadora Estatal de Normales del Estado de Oaxaca" (2012, p. 52).

\section{Reflexiones}

Sin duda, analizar la producción sobre un campo de conocimiento permite organizar cartografias sobre su configuración, además de establecer las correspondencias entre las formas de producción de saber local y las orientaciones que circulan a nivel internacional. El saber producido alrededor de lo que agrupa la denominación de escritura de sí se ha manufacturado como oposición a los grandes paradigmas del siglo pasado que tendían a anular las voces de los sujetos particulares. El saber biográfico se instituye como un giro hacia el sujeto concreto. En las ciencias sociales, su génesis se localiza en los trabajos abanderados desde la perspectiva del interaccionismo simbólico por Thomas y Znaniecki [1918/1927] (The Polish Peasant in Europe and America, 1996). Otras tradiciones entrarán al embate para abonar en la fundamentación del campo biográfico: fenomenologías, hermenéuticas y pragmatismos habría lugar para los existencialismos-.

Los textos confeccionados en la década que analizamos exponen sus filias y fobias. Combinan perspectivas metodológicas. En el terreno encuentran filones o vetas que abonan a los estilos distintivos de reflexión y a los ob- jetos, problemas o preguntas planteados por los investigadores en el camino de la indagación que han seleccionado. En el hit parade de la filiación están los argumentos ligados a Bourdieu, Berger y Luckman, Goffman, Garfinkel, Shutz, Schütze, Elder, Heller, Goodson, Ricoeur, Dubar, Halbwachs, Bolivar. Algunos de ellos fundadores de los barruntos que organizan -en las ciencias sociales- la tradición cualitativa sobre las que se asientan las argumentaciones del campo biográfico. Otras deliberaciones se desprenden de autores que han continuado el campo o de los que han puesto en juego argumentaciones ligadas a investigaciones concretas y que, en el trayecto, han elaborado nociones ad hoc.

La traducción de nociones amplias, o de principios argumentativos de las tradiciones mencionadas a objetos concretos de indagación, ha llevado a la edificación de razonamientos particulares alrededor de los sujetos o poblaciones indagadas, previamente seleccionadas. En ese sentido, se localizan elaboraciones que abonan a un mestizaje fructífero o a producciones bizarres que sostienen sus elucidaciones - dualistas o deterministas - contra los cuales bregó el punto de vista cualitativo. Los que trabajamos en el campo tendríamos que colocar mayor vigilancia a nuestros trabajos. Sin duda, se requiere mayor vigilancia epistemológica para los que hemos asumido la escritura de sí como ámbito de producción académica y sobre los cuales organizamos trayectos de formación para incluir a sucedáneos en el campo auto-bio-graphie (GUSDORF, 1991).

Las producciones relatadas se han adherido -en sus tesis- a los diversos giros que desde el siglo pasado impactan las argumentaciones, deliberaciones u objetos de indagación. Un tesoro lingüístico acompaña a cada posición. El giro a lo cotidiano nos orienta a localizar a las interacciones sociales del día a día; el giro lingüístico da nuevos contenidos 
a la idea de sujeto; el hermenéutico va de la mano de la comprensión y del círculo hermenéutico; el giro pragmático nos induce a pensar la definición de la situación de la acción social; el giro narrativo sostiene que la vida se vive como historia; el giro afectivo asume la sociedad afectiva como su égida. No todos los giros han provocado el sismo previsto, pero de todos ha abrevado el campo biográfico en las ciencias sociales. Este movimiento tiene variadas deliberaciones, acompañado de tradiciones lingüísticas propias: inglés, francés, alemán; unidas en los textos dan pie a nuevas orientaciones y variados objetos de trabajo.

Cabe particularizar el especial acento que en el contexto mexicano se da a lo biográfico. La noción de trayectoria merece su biografía particular y está por escribirse. Una referencia central es el texto de 1991 elaborado por Riemann y Schütze (en lengua alemana, Schütze se ha dedicado a reflexionar sobre los asuntos biográficos. La traducción de su obra resultará altamente relevante). Los autores hacen una breve reseña de la emergencia de la noción de trayectoria en los trabajos de las primeras camadas de investigadores ligados al interaccionismo simbólico. La cuna de la noción de trayectoria parece ser La escuela de Chicago. Los investigadores estuvieron atentos al sufrimiento de los sujetos y querían dar cuenta del proceso a través de nociones como carrera o pasaje de estatus, pero advirtieron la debilidad de éstos para dar cuenta de los intríngulis entre lo externo y lo individual. La noción de trayectoria viene a ser el eslabón. Para Riemann y Schütze "Order in life is mobilized by institutional expectation patterns and by stretched-out biographical action schemes. Institutional expectation patterns refer to the normative principle of biography, and biographical action schemes to its intentional principle" (1991, p. 339). De ahí el paso a la propuesta de Bourdieu -autor que desata diversas pasiones entre los académi- cos- que resuena en varios de los documentos expuestos en este texto, dice "la noción de trayectoria como serie de las posiciones sucesivamente ocupadas por un mismo agente (o un mismo grupo) en un espacio, en sí mismo en movimiento, y sometido a incesantes transformaciones" (1997, p.82). De esta caracterización se forman seguidores y se cohesionan objetores. Además, la literatura en el campo bio adosa otras nociones o las toma como sinónimos: itinerarios, travesías, recorridos.

De lo anterior se desprende un tema a deliberar: ¿hablamos de lo mismo cuando utilizamos palabras diferentes y recurrimos a autores de diversas tradiciones?, ¿están en la misma sintonía -al interior de las perspectivas fenomenológicas, hermenéuticas, pragmáticas o interaccionistas - las historias de vida, los relatos de vida, los testimonios, las trayectorias, los itinerarios o travesías? En el mismo orden de cuestionamientos está el epítome de lo biográfico: ¿son equiparables -desde distintas perspectivas- la vivencia, la experiencia, la experiencia vivida, el acontecimiento, el evento crítico?

Vale una mirada a las consideraciones mostradas acerca del sujeto en las indagaciones. En los textos existe una idea del sujeto que va desde un sujeto activo, constructor, a un sujeto constreñido por su mundo social y reproductor de las condiciones existentes, a pesar de que se apuntale la existencia de salidas posibles a su mundo de vida. Además, los textos deliberan sobre los sujetos o población a partir de la cual confeccionan los datos y, en general, lo denominan como sujeto colaborador.

Entre investigador (patrocinado ya sea por su institución o por resultar victorioso en la convocatoria de una agencia con un monto de dinero) y la población (objeto de los escrutinios de) existe asimetría en todos los planos. En los escritos hay un movimiento, reconocimiento de la diferencia o elaboración de mati- 
ces que, bajo la idea de colaboración, diluyen la asimetría. De informante a colaborador, sin los rendimientos que para los académicos tiene la indagación: puntajes en sistemas de becas, títulos académicos, reconocimiento social o simbólico en el instituido mercado académico. En cambio, la población lo que posee es la experiencia -relatada por otro-.

Un punto más para examinar es el tipo de estrategia -o el bricolaje de ellas- que se arrebujan bajo argumentaciones que oponen lo cualitativo versus lo cuantitativo y que se unen bajo la consideración de "metodologías mixtas". ¿Hay algún número en las indagaciones que se coloque sin que él mismo ya sea una cualidad del objeto? ¿Este tipo de consideraciones no son la reivindicación del dualismo contra el cual se oponen los fundadores del pensamiento cualitativo actual? Habrá que hacer un balance de pérdidas y ganancias que hemos tenido con el uso de los manuales de investigación en ciencias sociales que han perdido la veta de cogitación filosófica y que no dejan de apostar, en los hechos, por una visión empírico-analítica bajo la intención de "complementar" la lectura de la realidad.

Antes de finalizar, el lenguaje que utilizamos en la descripción de lo cualitativo es un síntoma. Utilizamos metáforas que ponen en jaque nuestra adhesión epistemológica: nuestra perspectiva muestra cómo lo externo e interno actúan en el campo educativo (expresión que reivindica una posición dualista); el sujeto es portador de experiencias (da la idea de que las experiencias y el sujeto están separadas, las experiencias vienen al lado, se olvida que el sujeto es experiencia); el sujeto y la institución (el sujeto ya es institución). Estamos a la víspera de elaborar nuevas metáforas para dar cuenta del sujeto sin oponer subjetividad a objetividad. Las metáforas de la intersubjetividad están por venir y superar nuestra herencia dualista.
Sin duda, llama la atención sobre la aprehensión de lo cualitativo como paradigma, modo de explicación, perspectiva y la ausencia notable de dar cuenta de la implicación de los sujetos en el proceso indagador. En la confección del conocimiento en las ciencias sociales, en particular para el campo biográfico, valdría la pena "usar la subjetividad propia de toda observación como camino real hacia una objetividad auténtica, no ficticia" (DEVEREUX, 1977, p. 20). Para finalizar cabe recordar las palabras de Devereux:

No es el estudio del sujeto sino el del observador el que nos proporciona acceso a la esencia de la situación. Los datos de la ciencia son entonces triples:

1. El comportamiento del sujeto.

2. Los "trastornos" producidos por la existencia y las actividades observacionales del observador.

3. El comportamiento del observador: sus angustias, sus maniobras defensivas, su estrategia de investigación, sus "decisiones" (= su atribución de un significado a la observado). (DEVEREUX, 1977, p. 22)

\section{Referencias}

BECHER, Tony. Tribus y territorios académicos. La indagación intelectual y las culturas de las disciplinas. Barcelona: Gedisa, 2002.

DALLE, Pablo et al. Manual de metodología. Construcción del marco teórico, formulación de los objetivos y elección de la metodología. Buenos Aires: Consejo Latinoamericano de Ciencias Sociales, 2005.

DEVEREUX, George. De la ansiedad al método en las ciencias del comportamiento. Ciudad de México: Siglo XXI, 1977.

FOESSEL, Michaël. Pensar lo social: entre la fenomenología y hermenéutica. En: DELACROIX, C.; DOSSE, F.; GARCÍA, P. (Dirs.). Paul Ricoeur y las ciencias humanas. Buenos Aires: Nueva Visión, 2008. p. 29-43. 
FRANCO, Martha Josefina. Configuración de identidades estudiantiles. El caso de jóvenes de un bachillerato en un contexto migratorio. En: LANDESMAN, Monique. (Coord.). Instituciones educativas, trayectorias e identidades de sus sujetos. Ciudad de México: UNAM POSGRADO; Díaz de Santos, 2015. p. 37-66.

GARCÍA, Gloria Elizabeth. Mi hijo, lo mejor que me ha pasado. Una aproximación a los significados de las trayectorias sexuales reproductivas de madres adolescentes en contexto de pobreza. Ciudad de México: Imjuve; Sedesol, 2016.

GONZÁLEZ, María Marcela. Transgrediendo la razón moderna. Aportes metodológicos para analizar las relaciones entre contexto y trayectorias de investigadoras educativas mexicanas. En: LANDESMAN, Monique. (Coord.). Instituciones educativas, trayectorias e identidades de sus sujetos. Ciudad de México: UNAM POSGRADO; Díaz de Santos. 2015. p. 179-220.

GÜEMES, Raquel. Trayectorias formativas e historias institucionales en la configuración de identidad. El caso de maestros de educación especial. En: LANDESMAN, Monique. (Coord.). Instituciones educativas, trayectorias e identidades de sus sujetos. Ciudad de México: UNAM POSGRADO; Díaz de Santos. 2015. p. 97-126.

GUSDORF, George. Auto-Bio-Graphie. Lignes de vie 2. París: Odile Jacob, 1991.

HICKMAN, Hortensia. Trayectorias académicas de fundadores y la institucionalización de la carrera de Psicología de la Universidad Veracruzana en Xalapa. En: LANDESMAN, Monique. (Coord.). Instituciones educativas, trayectorias e identidades de sus sujetos. Ciudad de México: UNAM POSGRADO; Díaz de Santos. 2015. p. 261-292.

IZQUIERDO, Isabel. Identidades e inmigración: los científicos soviéticos en los espacios mexicanos de investigación y docencia. En: LANDESMAN, Monique. (Coord.). Instituciones educativas, trayectorias e identidades de sus sujetos. Ciudad de México: UNAM POSGRADO; Díaz de Santos. 2015. p. 127-145.

JIMÉNEZ, Mariela Sonia. La construcción del estado del arte en la formación para la investigación en el posgrado en educación. En: PACHECHO, Teresa; DÍAZ
BARRIGA, Ángel. (Coords.). El posgrado en educación en México. Ciudad de México: Universidad Nacional Autónoma de México, 2009. p. 123-151.

MERINO, María del Carmen; RAMÍREZ, Teresita. La construcción subjetiva de la identidad en las autobiografias de estudiantes adolescentes. En: GUZMÁN, Carlota; SAUCEDO, Claudia. (Coords.). La voz de los estudiantes. Experiencias en torno a la escuela. Ciudad de México: UNAM; Pomares, 2007. p. $171-193$

MONTIEL, María Araceli. Vínculos, transferencias y deseo de saber. Reconstrucción de trayectorias académicas de prestigio: tres casos de la UNAM. Ciudad de México: ANUIES, 2014.

NAVARRO, Claudia. La antesala del ascenso a la dirección. Trayectorias de subdirectores de secundarias mexicanas. Ciudad de México: COMIE, 2015.

ORTIZ, Pedro; SERRANO, José Antonio. (Coords.). Formación de profesionales de la educación: sujetos, reflexión y producción de saber. Iguala: PEFEN SEP; CAM-Iguala; UPN Teloloapan SEP, 2016.

PERALES, Felipe de Jesús; JIMÉNEZ, Luz. Relevancia de las historias de vida de las y los docentes en la investigación cualitativa. En: SERRANO, José Antonio; RAMOS, Juan Mario. (Coords.). Trayectorias: biografias y prácticas. Ciudad de México: Universidad Pedagógica Nacional, 2011. p. 137-153.

QUEZADA, Margarita de Jesús. La escuela como formadora de identidades socioterritoriales. En: LANDESMAN, Monique. (Coords.). Instituciones educativas, trayectorias e identidades de sus sujetos. Ciudad de México: UNAM POSGRADO; Díaz de Santos, 2015. p. 67-96.

RAMOS, Juan Mario. Socialización en la educación básica: familia e imagen del maestro. En: BRAGANÇA, Inês Ferreira de Souza; ABRAHÃO, Maria Helena Menna Barreto; FERREIRA, Márcia Santos. (Orgs.). Perspectivas epistêmico-metodológicas da pesquisa (auto)biográfica. Curitiba: CRV, 2016. p. 283-300.

RAMOS, Juan Mario; SERRANO, José Antonio. La escritura autobiográfica de jóvenes y adultos en educación superior. Revista Brasileira de Educação de Jovens e Adultos, v. 5, n. 10, p. 77-89, 2017. 
RIEMANN, Gerhard; SCHÜTZE, Fritz. Trajectory as a basic theoretical concept for analyzing suffering and disorderly social processes. En: MAINES, David R. (Ed.). Social organization and social process: essays in honor of Anselm Strauss. New York: Gruyter, 1991. p. 333-357. Disponible en: <http://nbn-resolving.de/urn:nbn:de:0168-ssoar-7214>. Aceso en: 23 maio 2018.

RIVERA. Lucía. Aportes teórico-metodológicos para el estudio de las prácticas en contextos institucionales: el caso de las supervisoras de educación preescolar. En: LANDESMAN, Monique. (Coord.). Instituciones educativas, trayectorias e identidades de sus sujetos. Ciudad de México: UNAM POSGRADO; Díaz de Santos. 2015. p. 293-324.

ROMO, Rosa Martha. La memoria en la reconstrucción de culturas académicas. Relatos de vida y arribo de la primera psicopedagoga a la Universidad Autónoma de Nuevo León. En: LANDESMAN, Monique. (Coord.). Instituciones educativas, trayectorias e identidades de sus sujetos. Ciudad de México: UNAM POSGRADO; Díaz de Santos; 2015. p. 149-178.

ROMO, Rosa Martha; CRUZ, Evangelina. Identidades profesionales e historia heredada en académicos universitarios. Perfiles Educativos, Instituto de Investigaciones sobre la Universidad y la Educación, UNAM, v. XXXVII, n. 149, p. 42-59, 2015.

RUEDA, Mario. Presentación de la Colección. La investigación educativa en México (1992-2002). En: WEISS, Eduardo. (Coord.). La investigación educativa en México, 1993-2001. Ciudad de México: Consejo Mexicano de Investigación Educativa, 2003. p. 3-15.

RUİZ, María Mercedes. La secundaría para trabajadores vista a través de sus estudiantes: voces de exclusión desde la otra educación. Ciudad de México: Instituto de Investigaciones sobre la Universidad y la Educación; UNAM, 2012.

SALAZAR, María de Lourdes; SÁNCHEZ, María de Lourdes. Narrativas biográficas y trayectorias profesionales de educadoras del Gobierno del Distrito Federal, mujeres, madres, maestras. Ciudad de México: UPN, 2016.

SAUCEDO, Claudia. La importancia de la escuela en las experiencias de vida de los estudiantes: su valoración retrospectiva a través de relatos de vida. En: GUZMÁN, Carlota; SAUCEDO, Claudia. (Coords.). La voz de los estudiantes. Experiencias en torno a la escuela. Ciudad de México: UNAM; Pomares, 2007. p. 23-43.

SERRANO, José Antonio. Innovar a partir de la reflexión de la práctica educativa. En: Secretaría de Educación Pública. Cultivar la innovación. Hacia una cultura de la innovación. Ciudad de México: Secretaría de Educación Pública, 2009. Disponible en: <http:// biblio.upmx.mx/textos/21944.pdf>. Aceso en: 25 maio 2018.

SERRANO, José Antonio. Trayectoria(s) en la enseñanza de la teoría educativa y la docencia reflexiva. En: SERRANO, J. A.; RAMOS, J. M. (Coords.). Trayectorias: biografias y prácticas. Ciudad de México: Universidad Pedagógica Nacional, 2011. p. 123-135.

SERRANO, José Antonio. Periplos e inquietudes en la elaboración de trayectorias biográficas. Revista Educación, Universidad Nacional de Mar del Plata, Facultad de Humanidades, año 3, n. 4, p. 101-114, 2012a.

SERRANO, José Antonio. Indagación y narratividad en educación: intervenir e investigar. En: ABRAHÃO, María Helena; PASSEGGI, María da Conceição. (Orgs.). Dimensões epistemológicas e metodológicas da pesquisa (auto)biográfica. Tomo I. Natal: EDUFRN; Salvador: EDUNEB; Porto Alegre: EDIPUCRS; CNPQ, 2012b. p. 115-137.

SERRANO, José Antonio. Experiencia, narrativitat i formació. Quaderns d'educació contínua, del Centre de Recuros i Educació Contínua, Valencia, v. 28, p. 5-21, 2013.

SERRANO, José Antonio. Develar el saber de los docentes en las narrativas (auto)biográficas. En: ABRAHÃO, Maria Helena Menna Barreto; BRAGANÇA, Inês Ferreira de Souza; ARAÚJO, Mairce da Silva. (Orgs.). Pesquisa (auto)biográfica, fontes e questões. Curitiba: CRV, 2014. p. 197-214.

SERRANO, José Antonio. La educación básica: la experiencia en el oficio en el ser estudiante. En: PERIN P.; CUNHA J. L. da.; MACIEL, L. A. (Orgs.). Experiências 
formativas e práticas de iniciação à docência. Curitiba: CRV, 2016a. p. 149-164.

SERRANO, José Antonio. La entrevista: entre zozobras y salir victorioso en la indagación. En: SOUZA, Elizeu. (Org.). (Auto)biografías e documentação narrativa. Redes de pesquisa e formação. Salvador: EDUFBA, 2016b. p. 261-284.

SERRANO, José Antonio; FURLÁN, Alfredo. Posiciones en la configuración del campo de formación de docentes en educación superior. En: SERRANO, J. A.; RAMOS, J. M. (Coords.). Trayectorias: biografias y prácticas. Ciudad de México: Universidad Pedagógica Nacional, 2011. p. 73-121.

SERRANO, José Antonio et al. Lo biográfico, pases de estafeta y diálogo inter(intra)disciplinario: experiencia y narratividad. Revista Inter-Legere, Programa de Pós-Graduação em Ciências Sociais da UFRN, n. 16, p. 140-160, jan./jun. 2015.

SERRANO, José Antonio; RAMOS, Juan Mario. Boceto del espacio biográfico-educativo en México (200032013). Revista Mexicana de Investigación Educativa, v. XIX, n. 62, p. 831-858, 2014.

SERRANO, José Antonio; RAMOS, Juan Mario. El sujeto: saber, institución y cultura. En: ORTIZ, Pedro; SERRANO, José Antonio. (Coords.). Formación de profesionales de la educación: sujetos, reflexión y producción de saber. Iguala: PEFEN SEP; CAM Iguala; UPN Teloloapan SEP, 2016. p. 15-32.

SERRANO, José Antonio; RAMOS, Juan Mario. Narrar a vida: deliberações no campo biográfico. En: MARTINS, Raimundo; TOURINHO, Irene; SOUZA, Elizeu. (Orgs). Pesquisa narrativa. Interfaces entre história de vida, arte e educação. Santa Maria: Editora da UFSM, 2017a. p. 75-97.

SERRANO, José Antonio; RAMOS, Juan Mario. Ecos desde la perspectiva narrativa en la formación de profesionales de la educación. Revista del Instituto de investigaciones en Ciencias de la Educación, Universidad de Buenos Aires, FFyL, 2017b. (en prensa).

TORIZ, Acacia. Transformaciones de la profesión docente como una institución y malestar en la escuela secundaria pública. Trayecto metodológico. En: LANDESMAN, Monique. (Coord.). Instituciones educativas, trayectorias e identidades de sus sujetos. Ciudad de México: UNAM POSGRADO; Díaz de Santos, 2015. p. 325-355.

TORRES, Rosa María. Advenimiento y construcción de la narrativa autobiográfica. En: RUIZ, Dalia. (Coord.). Cuerpos académicos. Vía alterna hacia la investigación. Ciudad de México: Universidad Pedagógica Nacional, 2011. p. 249-261.

TORRES, Rosa María. Los tiempos y espacios en el campo: factores sustantivos para los trayectos profesionales de docentes. En: SOUZA, Elizeu. (Org.). Educação e ruralidades, memórias e narrativas (auto)biográficas. Salvador: EDUFBA, 2012. p. 51-68.

VELÁZQUEZ, Luz María. Preparatorianos: trayectorias y experiencias en la escuela. En: GUZMÁN, Carlota; SAUCEDO, Claudia. (Coords.). La voz de los estudiantes. Experiencias en torno a la escuela. Ciudad de México: UNAM; Pomares, 2007. p. 44-68.

ZAMORA, Antonio. Trayectorias profesionales anudadas a historias socio-institucionales: el caso de académicos de la UPN. En: LANDESMAN, Monique. (Coord.). Instituciones educativas, trayectorias e identidades de sus sujetos. Ciudad de México: UNAM POSGRADO; Díaz de Santos, 2015. p. 221-260.

Recebido em: 11.02.2018 Aprovado em: 11.08 .2018

José Antonio Serrano Castañeda Doctor en Pedagogía por la Universidad de Barcelona. Profesor Titular, SNI-1 Conacyt y profesor PRODEP. Integrante del cuerpo académico consolidado Prácticas institucionales y constitución del sujeto de la educación (CAC PICSE). Es responsable de la Red de investigación dedicada a la reflexión sobre lo biográfico y la narrativa en educación SPECE (Sujetos y prácticas educativas en contextos escolares). Ha participado en diferentes proyectos de investigación, así como de formación de docentes en el sistema educativo mexicano. Ha publicado diversos artículos sobre teoría educativa, curriculum, formación de docentes, alfabetización académica y el campo biográfico-narrativo. e-mail: jserrano@g.upn.mx 
Juan Mario Ramos Morales Doctor en Ciencias Administrativas por el Instituto Tecnológico y de Estudios Superiores de Monterrey, Campus Ciudad de México. Profesor Titular, SNI-1 Conacyt y profesor PRODEP. Integrante del cuerpo académico consolidado Prácticas institucionales y constitución del sujeto de la educación (CAC PICSE) y de la Red SPECE (Sujetos y prácticas educativas en contextos escolares). Es responsable de la Maestría en Desarrollo Educativo, programa consolidado en las políticas de CONACYT. Ha participado en diferentes proyectos de investigación. Ha publicado diversos artículos sobre el campo biográfico-narrativo en educación.e-mail: juanmarioramos@gmail.com

Universidad Pedagógica Nacional. Carretera al Ajusco 24, Col. Héroes de Padierna, 06470, Tlalpan, Ciudad de México. (52) 56309700, ext. 1145, 1415 